\title{
Towards models with a unified dynamical mechanism for elementary particle masses
}

\author{
Roberto Frezzotti* \\ Dipartimento di Fisica, Università di Roma "Tor Vergata" and INFN, Sezione di Roma Tor \\ Vergata - Rome, Italy \\ E-mail: roberto.frezzotti@roma2.infn.it
}

\section{Giancarlo Rossi}

Dipartimento di Fisica, Università di Roma "Tor Vergata", and INFN, Sezione di Roma Tor Vergata, and Centro Fermi, Museo Storico della Fisica - Rome, Italy

E-mail: rossigaroma2.infn.it

\begin{abstract}
Numerical evidence for a new dynamical mechanism of elementary particle mass generation has been found by lattice simulation in a simple, yet highly non-trivial SU(3) gauge model where a $\mathrm{SU}(2)$ doublet of strongly interacting fermions is coupled to a complex scalar field doublet via a Yukawa and a Wilson-like term. We point out that if, as a next step towards the construction of a realistic beyond-the-Standard-Model model, weak interactions are introduced, then also weak bosons get a mass by the very same non-perturbative mechanism. In this scenario fermion mass hierarchy can be naturally understood owing to the peculiar gauge coupling dependence of the non-perturbatively generated masses. Hence, if the phenomenological value of the mass of the top quark or the weak bosons has to be reproduced, the RGI scale of the theory must be much larger than $\Lambda_{Q C D}$. This feature hints at the existence of new strong interactions and particles at a scale $\Lambda_{T}$ of a few TeV. In such a speculative framework the electroweak scale can be derived from the basic scale $\Lambda_{T}$ and the Higgs boson should arise as a bound state in the $W W+Z Z$ channel.
\end{abstract}

The 36th Annual International Symposium on Lattice Field Theory - LATTICE2018

22-28 July, 2018

Michigan State University, East Lansing, Michigan, USA.

\footnotetext{
* Speaker.
} 


\section{Elementary particle masses from a "non-perturbative anomaly"}

The Standard Model (SM) of elementary particles, in spite of its very impressive successes, is widely believed to be only an effective low energy theory as it neither accounts for dark matter and the quantum aspects of gravity nor provides enough CP-violation for baryogenesis. Moreover, the SM is by construction unable to shed light on the puzzling problems of EW scale naturalness [1] and fermion mass hierarchy [2]. Apart from these still open problems, the authors of Ref. [3] pointed out that, if some dynamical mechanism involving non-SM interactions gives rise to the mass of the known elementary fermions, the same mechanism also yields massive $W^{ \pm}, Z^{0}$ bosons and a composite Higgs boson in the $W^{+} W^{-}, Z^{0} Z^{0}$, and/or $t \bar{t}$ channel.

Following the remark of Ref. [3] and with the aim of solving the general problems of fermion mass hierarchy and EW scale naturalness, as well as certain problems [4] of Ref. [3] with a proper definition of the composite Higgs framework, in Ref. [5] a new mechanism for the dynamical generation of elementary fermion masses was advocated. This mechanism is conjectured to be at work in non-Abelian gauge models with fermions and scalars where 1) (as usual) chiral transformations acting on fermions and scalars are exact symmetries, but 2) (deviating from common assumptions) purely fermionic chiral symmetries undergo a hard breaking at the UV cutoff scale. When bare parameters are "naturally" tuned so as to minimize fermion chiral breaking, in the effective Lagrangian (EL) [6] no Yukawa term occurs, but operators of non-perturbative origin that violate fermion chiral symmetries, among which a fermion mass term, are expected to appear, if the scalar potential is such that the theory lives in its Nambu-Goldstone (NG) phase.

In the proceeding contribution [7] to Lattice 2018 convincing numerical evidence was provided that the mass generation mechanism of Ref. [5] is indeed realized in the simplest ("toy", yet highly non-trivial) $d=4$ lattice gauge model where this phenomenon could take place. To comply with the requirements 1) and 2) above the model considered in Ref. [7] contains

- an SU(3) gauge field, $A_{\mu}^{c}(c=1,2, \ldots, 8)$, with bare (renormalized) coupling $g_{0}\left(g_{S}\right)$,

- one Dirac fermion doublet, $Q=(u, d)^{T}$, transforming as a colour triplet under $\mathrm{SU}(3)$,

- one complex scalar doublet, $\varphi=\left(\varphi_{0}+i \varphi_{3},-\varphi_{2}+i \varphi_{1}\right)^{T}$, invariant under SU(3). Adopting the $2 \times 2$ matrix notation $\Phi=\left[\varphi \mid-i \tau^{2} \varphi^{*}\right]$, the toy model Lagrangian, $\mathscr{L}_{\text {toy }}(Q, A, \Phi)$, takes the form

$$
\mathscr{L}_{\text {toy }}=\mathscr{L}_{k}(Q, A, \Phi)+\mathscr{V}(\Phi)+\mathscr{L}_{W i l}(Q, A, \Phi)+\mathscr{L}_{\text {Yuk }}(Q, \Phi),
$$

with $\mathscr{L}_{k}$ and $\mathscr{V}$ representing standard kinetic terms and the scalar potential. The model has UV cutoff $\Lambda_{U V} \sim b^{-1}$ and a renormalization group invariant (RGI) dynamical scale $\Lambda_{S}$. Its Lagrangian includes also a Yukawa term, $\mathscr{L}_{\text {Yuk }}(Q, \Phi)=\eta\left(\bar{Q}_{L} \Phi Q_{R}+\bar{Q}_{R} \Phi^{\dagger} Q_{L}\right)$, and a term $\mathscr{L}_{W i l}(Q, A, \Phi)=$ $\frac{b^{2}}{2} \rho\left(\bar{Q}_{L} \overleftarrow{\mathscr{D}}_{\mu} \Phi \mathscr{D}_{\mu} Q_{R}+\bar{Q}_{R} \overleftarrow{\mathscr{D}}_{\mu} \Phi^{\dagger} \mathscr{D}_{\mu} Q_{L}\right)$. The latter, being a $\Lambda_{U V}^{-2} \times d=6$ operator, leaves the model power-counting renormalizable [5], exactly like it happens for the Wilson term in lattice QCD [8, 9], but induces a hard breaking of the purely fermionic chiral symmetries. Among other symmetries, the Lagrangian (1.1) is invariant under the global transformations $\left(\Omega_{L / R} \in \mathrm{SU}(2)\right)$

$$
\begin{aligned}
& \chi_{L} \times \chi_{R}=\left[\tilde{\chi}_{L} \times\left(\Phi \rightarrow \Omega_{L} \Phi\right)\right] \times\left[\tilde{\chi}_{R} \times\left(\Phi \rightarrow \Phi \Omega_{R}^{\dagger}\right)\right], \\
& \tilde{\chi}_{L / R}: Q_{L / R} \rightarrow \Omega_{L / R} Q_{L / R}, \quad \bar{Q}_{L / R} \rightarrow \bar{Q}_{L / R} \Omega_{L / R}^{\dagger} .
\end{aligned}
$$

A (divergent) fermion mass term $\sim \Lambda_{U V}\left(\bar{Q}_{L} Q_{R}+\right.$ h.c.), being $\chi_{L} \times \chi_{R}$ variant, is not generated. 
Although the Lagrangian (1.1) is not invariant under the purely fermionic chiral transformations $\tilde{\chi}_{L} \times \tilde{\chi}_{R}$, a critical value of the Yukawa coupling, $\eta_{c r}$, exists at which the effective Yukawa term vanishes [5, 7]. In the phase with positive renormalized squared scalar mass, $\hat{\mu}_{\phi}^{2}=Z_{\Phi^{\dagger} \Phi}^{-1}\left(\mu_{0}^{2}-\right.$ $\left.\mu_{c r}^{2}\right)>0$, where the $\chi_{L} \times \chi_{R}$ symmetry is realized $\grave{a}$ la Wigner, at $\eta=\eta_{c r}$ the transformations of $\tilde{\chi}_{L} \times \tilde{\chi}_{R}$ become approximate (up to corrections $\mathrm{O}\left(\Lambda_{U V}^{-2}\right)$ ) symmetries.

In the phase where $\hat{\mu}_{\phi}^{2}<0$, due to the double-well shape of $\mathscr{V}(\Phi),\left\langle: \Phi^{\dagger} \Phi:\right\rangle=v^{2} 11, v \neq 0$ and the $\chi_{L} \times \chi_{R}$ symmetry is realized à la NG, i.e. spontaneously broken down to $\mathrm{SU}(2)_{V}$. Moreover, owing to $v \neq 0$, at $\eta=\eta_{c r}$ the residual $\mathrm{O}\left(\Lambda_{U V}^{-2}\right)$ and $\tilde{\chi}_{L} \times \tilde{\chi}_{R}$ violating action terms polarize the vacuum that is degenerate as a result of the dynamical $\tilde{\chi}_{L} \times \tilde{\chi}_{R}$ spontaneous breaking ensuing from strong interactions. In this situation, at $\eta=\eta_{c r}$ the $d \leq 4$ piece of the EL is conjectured [5] to read

$\left.\Gamma_{4}^{N G}\right|_{\eta_{c r}}=c_{2} \Lambda_{S}^{2} \operatorname{tr}\left(\partial_{\mu} U^{\dagger} \partial_{\mu} U\right)+c_{1} \Lambda_{S}\left[\bar{Q}_{L} U Q_{R}+\right.$ h.c. $]+\tilde{c} \Lambda_{S} R \operatorname{tr}\left(\partial_{\mu} U^{\dagger} \partial_{\mu} U\right)+\Gamma_{k}+\hat{\mathscr{V}}+\mathrm{O}\left(\Lambda_{S}^{2} v^{-2}\right)$,

with effective scalar potential $\hat{\mathscr{V}}$ and kinetic terms $\Gamma_{k}=\frac{1}{4}(F F)+\sum_{X=L, R} \bar{Q}_{X} \not Q_{X}+\frac{1}{2} \operatorname{tr}\left[\partial_{\mu} \Phi^{\dagger} \partial_{\mu} \Phi\right]$. Here, owing to $v \neq 0$, the effective scalar field is conveniently rewritten in terms of Goldstone $\left(\zeta_{1,2,3}\right)$ and massive $\left(\zeta_{0}\right)$ scalar fields: $\Phi=R U, \quad R=\left(v+\zeta_{0}\right), \quad U=\exp \left[i v^{-1} \tau^{k} \zeta_{k}\right]$, where $U$ is a dimensionless effective field transforming as $U \rightarrow \Omega_{L} U \Omega_{R}^{\dagger}$ under $\chi_{L} \times \chi_{R}$. It represents the exponential Goldstone boson map and is well defined only if $v \neq 0$. In Eq. (1.4) the term $\propto c_{1}$ describes non-perturbative (NP) breaking of $\tilde{\chi}_{L} \times \tilde{\chi}_{R}$ and provides an effective mass for the fermion fields. In fact, expanding $U$ around the identity, one gets $c_{1} \Lambda_{S}\left[\bar{Q}_{L} U Q_{R}+\bar{Q}_{R} U^{\dagger} Q_{L}\right]=$ $c_{1} \Lambda_{S} \bar{Q} Q[1+\mathrm{O}(\zeta / v)]$, i.e. a fermion mass term plus a host of more complicated, non-polynomial $\bar{Q}-\zeta_{1,2,3}$ particles $-Q$ interactions. The coefficient $c_{1}$ in Eq. (1.4) has been argued in Ref. [5] to be an $\mathrm{O}\left(g_{S}^{4}\right)$, odd function of $\rho$, with its $\lambda_{0}$ dependence arising only at high loop orders. As for its dependence on the scalar squared mass, $c_{1}$ is expected to stay finite in the limit $-\hat{\mu}_{\phi}^{2} \gg \Lambda_{S}^{2}$ (which is of phenomenological interest, see Refs. [5]) and to be non-zero only for $\hat{\mu}_{\phi}^{2}<0$.

A proper understanding of all the NP terms in the EL expression (1.4) requires considering the natural extension of $\tilde{\chi}_{L} \times \tilde{\chi}_{R}$ symmetry in the presence of weak interactions [10] - see Sect. 2. The form of $\left.\Gamma_{4}^{N G}\right|_{\eta_{c r}}$ implies that the renormalized $\tilde{\chi}_{L} \times \tilde{\chi}_{R}$ Schwinger-Dyson equations (SDE) contain in their r.h.s. $\tilde{\chi}$-violating NP terms which, if non-zero, must be RGI, as the 1.h.s. of the SDE is. Indeed at $\eta_{c r}$ the $\tilde{\chi}_{L} \times \tilde{\chi}_{R}$ currents have (independently of $\hat{\mu}_{\phi}^{2}$ ) zero anomalous dimension [5]. The full NG phase EL, $\Gamma^{N G} \supset \Gamma_{4}^{N G}$, contains of course an infinite set of local terms of arbitrarily high dimension, among which the RGI operators of NP origin that violate the approximate $\tilde{\chi}_{L} \times \tilde{\chi}_{R}$ symmetry. This phenomenon will be referred to as a "NP anomaly" in the $\tilde{\chi}$ symmetry restoration.

\section{Toy model with one strong and one weak interaction}

We consider here an extension of the toy model (1.1) including strong (vector SU(3): coupling $g_{S}$ ) and weak (chiral SU(2) $)_{L}$ : coupling $g_{W}$ ) gauge interactions plus a minimal Dirac fermion content that is enough to avoid Witten's global SU(2) anomaly [11]: a SU(3)-triplet field $Q$ and a $\mathrm{SU}(3)$-singlet field $N$, whose left-handed (right-handed) components are in the fundamental (trivial) representation of the weak $\mathrm{SU}(2)$ gauge group. Its classical Lagrangian takes thus the form

$$
\mathscr{L}_{\text {toy }+\mathrm{W}}=\mathscr{L}_{k}(Q, N, A, \Phi, W)+\mathscr{V}(\Phi)+\mathscr{L}_{W i l}(Q, N, A, \Phi, W)+\mathscr{L}_{\text {Yuk }}(Q, N, \Phi),
$$


where $\mathscr{V}(\Phi)$ is the potential of the scalar $\Phi$-field, $\mathscr{L}_{Y u k}(Q, N, \Phi)=\eta_{Q} \bar{Q}_{L} \Phi Q_{R}+\eta_{N} N_{L} \Phi N_{R}+$ h.c. is a standard Yukawa term, while the kinetic and the $\tilde{\chi}$-violating $d \geq 6$ terms read

$$
\begin{gathered}
\mathscr{L}_{k i n}=\frac{1}{4} F_{\mu \nu}^{A ; a} F_{\mu \nu}^{A ; a}+\frac{1}{4} F_{\mu \nu}^{W ; i} F_{\mu \nu}^{W ; i}+\bar{Q}_{L} \gamma_{\mu} \mathscr{D}_{\mu}^{A, W} Q_{L}+\bar{Q}_{R} \gamma_{\mu} \mathscr{D}_{\mu}^{A} Q_{R}+ \\
+\bar{N}_{L} \gamma_{\mu} \mathscr{D}_{\mu}^{W} N_{L}+\bar{N}_{R} \gamma_{\mu} \partial_{\mu} N_{R}+\frac{1}{2} \operatorname{tr}\left[\Phi^{\dagger} \overleftarrow{D}_{\mu}^{W} \mathscr{D}_{\mu}^{W} \Phi\right] \\
\mathscr{L}_{W i l}=\frac{1}{2 \Lambda_{U V}^{2}} \rho\left(\bar{Q}_{L} \overleftarrow{\mathscr{D}}_{\mu}^{A, W} \Phi \mathscr{D}_{\mu}^{A} Q_{R}+\bar{Q}_{R} \overleftarrow{\mathscr{D}}_{\mu}^{A} \Phi^{\dagger} \mathscr{D}_{\mu}^{A, W} Q_{L}+N_{L} \overleftarrow{\mathscr{D}}_{\mu}^{W} \Phi \partial_{\mu} N_{R}+\bar{N}_{R} \overleftarrow{\partial}_{\mu} \Phi^{\dagger} \mathscr{D}_{\mu}^{W} N_{L}\right)
\end{gathered}
$$

If the $W_{\mu}^{1,2,3}$ bosons transforms in the adjoint representation of $\mathrm{SU}(2)_{L}$ this model enjoys an exact $\mathrm{SU}(2)_{L} \times \mathrm{SU}(3)$ gauge symmetry, with the covariant derivatives acting on $f=Q, N$ given by e.g.

$$
\mathscr{D}_{\mu}^{A, W} f_{L}=\left(\partial_{\mu}-i \delta_{f, Q} g_{S} \lambda^{a} A_{\mu}^{a}-i g_{W} \frac{\tau^{i}}{2} W_{\mu}^{i}\right) f_{L}, \quad \bar{f}_{L} \overleftarrow{\mathscr{D}}_{\mu}^{A, W}=\bar{f}_{L}\left(\overleftarrow{\partial}_{\mu}+i \delta_{f, Q} g_{S} \lambda^{a} A_{\mu}^{a}+i g_{W} \frac{\tau^{i}}{2} W_{\mu}^{i}\right)
$$

Besides Lorentz, translation, time-reversal and CP symmetries, the model (2.1) enjoys the global $\mathrm{SU}(2)_{L} \times \mathrm{SU}(2)_{R}$ invariance $\left(\Omega_{L}\right.$ and $\Omega_{R}$ below are independent $\mathrm{SU}(2)$ matrices)

$$
\chi_{L} \equiv \tilde{\chi}_{L} \otimes \chi_{L}^{\Phi}, \quad \chi_{R} \equiv \tilde{\chi}_{R} \otimes \chi_{R}^{\Phi},
$$

where $\chi_{L, R}^{\Phi}$ acts only on scalars, $\chi_{L}^{\Phi}: \Phi \rightarrow \Omega_{L} \Phi, \quad \chi_{R}^{\Phi}: \Phi \rightarrow \Phi \Omega_{R}^{\dagger}$, while $\tilde{\chi}_{R}$ acts on right-handed fermions $f_{R}=Q_{R}, N_{R}$ and $\tilde{\chi}_{L}$ on left-handed fermions $f_{L}=Q_{L}, N_{L}$ and $W_{\mu}$ bosons (as necessary for invariance of the $Q_{L}$ and $N_{L}$ kinetic terms under $\chi_{L}$ )

$$
\begin{gathered}
f_{R} \rightarrow \Omega_{L} f_{R}, \quad \bar{f}_{R} \rightarrow \Omega_{R}^{\dagger} \bar{f}_{R}, \quad f \in\{Q, N\}, \\
f_{L} \rightarrow \Omega_{L} f_{L}, \quad \bar{f}_{L} \rightarrow \Omega_{L}^{\dagger} \bar{f}_{L}, \quad W_{\mu} \rightarrow \Omega_{L} W_{\mu} \Omega_{L}^{\dagger}, \quad f \in\{Q, N\} .
\end{gathered}
$$

Neither $\tilde{\chi}_{L, R}$ nor $\chi_{L, R}^{\Phi}$ transformations alone are symmetries of the model (2.1). For $g_{W} \neq 0$, not only $\mathscr{L}_{Y u k}$ and $\mathscr{L}_{W i l}$ but also the $Q_{L}$ and $N_{L}$ kinetic terms are not left invariant by $\tilde{\chi}_{L}$ transformations.

The study of the bare SDE of $\tilde{\chi}_{L}$ transformations shows that, owing to the symmetries of the model (2.1), the $\tilde{\chi}_{L}$-violating operators arising from $\mathscr{L}_{Y u k}$ and $\mathscr{L}_{W i l}$ and the fermion kinetic terms in $\mathscr{L}_{\text {toy }+W}$ mix only with two relevant $d=4$ operators, namely the $\tilde{\chi}_{L}$-variations of the Yukawa and the $\Phi$-kinetic term (the mixing coefficients are denoted below by $\bar{\eta}_{L}$ and $-\bar{\gamma}$ ). The renormalized form of the effective (isotriplet) $\tilde{\chi}_{L}-\mathrm{SDE}$ will thus read in synthetic operator notation

$$
\begin{aligned}
& Z_{\tilde{J}} \partial_{\mu} \tilde{J}_{\mu}^{L, i}=\left(\bar{\eta}_{L}-\eta\right) \sum_{f=Q, N}\left(\bar{f}_{L} \frac{\tau^{i}}{2} \Phi f_{R}-\text { h.c. }\right)+(1-\bar{\gamma}) \frac{i}{2} g_{W} \operatorname{tr}\left(\Phi^{\dagger}\left[\frac{\tau^{i}}{2}, W_{\mu}\right] \mathscr{D}_{\mu}^{W} \Phi+\text { h.c. }\right)+\ldots \\
& \tilde{J}_{\mu}^{L, i}=\sum_{f=Q, N}\left\{\bar{f}_{L} \gamma_{\mu} \frac{\tau^{i}}{2} f_{L}-\frac{b^{2}}{2} \rho\left(\bar{f}_{L} \frac{\tau^{i}}{2} \Phi \mathscr{D}_{\mu}^{A} f_{R}-\bar{f}_{R} \overleftarrow{\mathscr{D}}_{\mu}^{A} \Phi^{\dagger} \frac{\tau^{i}}{2} f_{L}\right)\right\}+i g_{W} \operatorname{tr}\left(\left[W_{v}, F_{\mu \nu}^{W}\right] \frac{\tau^{i}}{2}\right),
\end{aligned}
$$

where we omit irrelevant $\mathrm{O}\left(\Lambda_{U V}^{-2}\right)$ terms and ellipses stand for possible contributions from effective operators related to "NP anomalies", involving the field $U=\Phi / \sqrt{\Phi^{\dagger} \Phi}$ and to be discussed below. Eq. (2.7) implies that the condition of maximal restoration of the $\tilde{\chi}_{L}$ symmetry amounts to

$$
\eta_{c r}=\bar{\eta}_{L}\left(g_{s}, g_{W} ; \lambda_{0} ; \eta_{c r}, \rho_{c r}\right), \quad 1=\bar{\gamma}\left(g_{s}, g_{W} ; \lambda_{0} ; \eta_{c r}, \rho_{c r}\right)
$$


which is satisfied for suitable critical values, $\eta_{c r}$ and $\rho_{c r}$ of the bare parameters $\eta$ and $\rho$. Below we shall refer to the model (2.1) at $\eta_{c r}$ and $\rho_{c r}$ as to the "critical model".

The meaning of the condition of maximal $\tilde{\chi}_{L}$ symmetry restoration is particularly transparent in the Wigner phase of the model, where $\hat{\mu}_{\phi}^{2}>0$ and "NP anomalies" cannot occur because $v=0$ and the field $U=\Phi / \sqrt{\Phi^{\dagger} \Phi}$ entering their effective theory description is not defined. In this case the condition (2.8) makes the $\tilde{\chi}_{L}$-SDE (2.7) to look as a symmetry Ward identity, up to irrevant $\mathrm{O}\left(\Lambda_{U V}^{-2}\right)$ terms. As for the EL of the critical model, $\Gamma_{c r}^{W i g}$, this means that its $d \leq 4$ piece reads

$$
\Gamma_{4, c r}^{W i g} \equiv \Gamma_{4, c r}^{\hat{\mu}_{\phi}^{2}>0}=\frac{1}{4}\left[\left(F^{A} F^{A}\right)+\left(F^{W} F^{W}\right)\right]+\sum_{f=Q, N}\left[\bar{f}_{L} \mathscr{p}^{A, W} f_{L}+\bar{f}_{R} \not \mathscr{P}^{A} f_{R}\right]+\mathscr{V}_{\text {eff }}^{\hat{\mu}_{\phi}^{2}>0}[\Phi] .
$$

The absence of an effective Yukawa term in $\Gamma_{4, c r}^{W i g}$ implies that $\tilde{\chi}_{R}$ symmetry is recovered, too, while the cancellation of the effective $\Phi$-kinetic terms means that the elementary scalar field gets completely decoupled at all physical momentum scales (i.e. well below $\Lambda_{U V} \rightarrow \infty$ ).
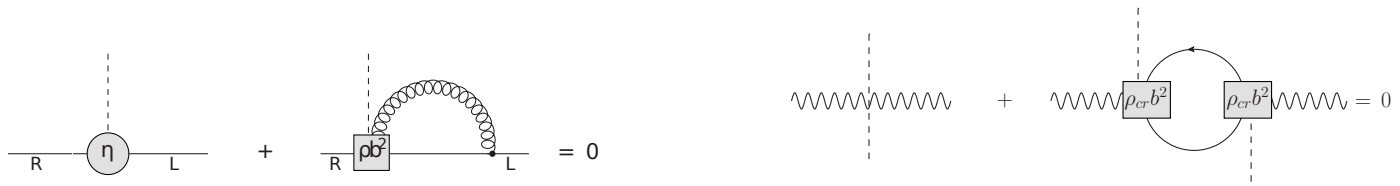

Figure 1: The vanishing in the critical model of the effective Yukawa vertex (left panel) and the effective $\Phi \Phi^{\dagger} W W$ vertex (right panel) is illustrated at the lowest non-trivial order of perturbation theory.

In the NG phase, where $\hat{\mu}_{\phi}^{2}<0$, the EL of the critical model still has vanishing effective Yukawa and $\Phi$-kinetic terms, but includes the terms describing the "NP anomalies" that are expected (known, according to the numerical evidence of Ref. [7] - see Sect. 1) to occur owing to $v>0$ and the fully realized spontaneous breaking of the approximate (restored) $\tilde{\chi}_{L} \times \tilde{\chi}_{R}$ symmetry:

$$
\Gamma_{c r}^{N G} \supset \Gamma_{4, c r}^{N G}, \quad \Gamma_{4, c r}^{N G}=C_{2} \Lambda_{S}^{2} \operatorname{tr}\left(\mathscr{D}_{\mu}^{W} U^{\dagger} \mathscr{D}_{\mu}^{W} U\right)+C_{1, Q} \Lambda_{S}\left[\bar{Q}_{L} U Q_{R}+\text { h.c. }\right]+\Gamma_{4, c r}^{\hat{\mu}_{\phi}^{2}<0},
$$

where $\Gamma_{4, c r}^{\hat{\mu}_{\phi}^{2}<0}$ is analogous to Eq. (2.9) but with $\hat{\mu}_{\phi}^{2}<0$. At this stage several remarks are in order. Remark I) $C_{1, N}=0$ because $N_{R}$ fermions are sterile, implying that $\mathscr{L}_{\text {toy }+\mathrm{W}}$ is invariant under the shift symmetry [12] $N_{R}(x) \rightarrow N_{R}(x)+c, \quad \bar{N}_{R}(x) \rightarrow \bar{N}_{R}(x)+\bar{c}$, with $c, \bar{c}$ Grassmann-number constants, which in turn forbids any mass term for the $N$ fields, including also $\Lambda_{S}\left[\bar{N}_{L} U N_{R}+\right.$ h.c. $]$.

Remark II) From the form of $\Gamma_{4, c r}^{N G}$ in Eq. (2.10) we see that $W_{\mu}$ and $Q$ fields get a dynamical mass,

$$
\left(M_{W}^{e f f}\right)^{2}=g_{W}^{2} C_{2} \Lambda_{S}^{2}, \quad m_{Q}^{e f f}=C_{1, Q} \Lambda_{S},
$$

which can be shown [10] to arise from a common NP mechanism where both $C_{2}$ and $C_{1}$ are $\ll 1$ due to multi-loop suppression. In fact, assuming the same kind of short distance NP vertex corrections that were conjectured in Ref. [5] (see there Eqs. (4.12)-(4.14) and Fig. 5), which explain the generation of the NP $Q$-fermion mass $m_{Q}^{\text {eff }}$ (see Sect. IV-C and Fig. 6 of Ref. [5]), one can understand/predict the occurrence of the $W_{\mu}$-boson mass term $\propto C_{2}$. The lowest loop order "diagram" relevant for $\left(M_{W}^{e f f}\right)^{2}$, which combines $\mathrm{O}\left(\Lambda_{U V}^{-2}\right) \tilde{\chi}$ violating vertices and the conjectured short distance NP vertices is shown in Fig. 2.

Remark III) In the NG phase of the critical model, owing to the "NP anomaly", the Goldstone boson modes of the elementary $\Phi$ are still coupled to fermions and $W_{\mu}$ bosons. The canonically 

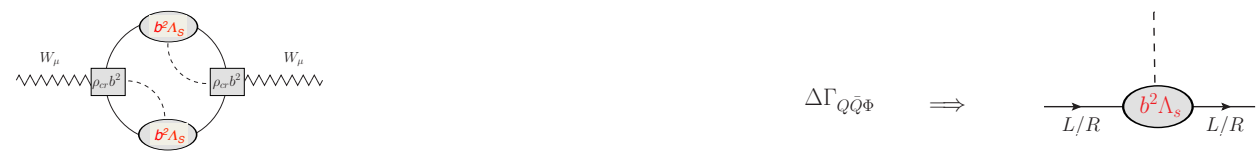

Figure 2: The lowest loop order "diagram" relevant for $\left(M_{W}^{e f f}\right)^{2}$ (left panel) and the short distance NP vertex $\Delta \Gamma_{Q \bar{Q} \Phi}$ (right panel). The vertices with a square $\propto b^{2} \rho_{c r} \equiv \Lambda_{U V}^{-2} \rho_{c r}$ come from the $\mathscr{L}_{W i l}$ term of the Lagrangian (2.1). Here and in Fig. 1 lines denote $Q$ fermions, wavy lines $W_{\mu}$ bosons, dashed lines $\Phi$ bosons.

normalized Goldstone boson fields $\zeta_{1,2,3}$ are hence given by $U=\Phi / \sqrt{\Phi^{\dagger} \Phi}=\exp \left[i \zeta_{j} \tau^{j} / \Lambda_{S} \sqrt{C_{2}}\right]$. From $\Gamma_{4, c r}^{N G}$ one sees that the $W W$-propagator comes out to be properly transverse owing to the contribution from effective vertices with one $W_{\mu}^{j}$ and one $\zeta_{j}$ field. In unitary gauge the Goldstone bosons are eaten up by the massive $W_{\mu}$ bosons - just as in the standard Higgs mechanism.

Remark IV) The non-Goldstone mode $\zeta_{0}$ in $|\Phi|=R=v+\zeta_{0}$ is instead an auxiliary and fully decoupled field in the critical model at all physical momentum scales, because of the absence in $\Gamma_{4, c r}^{N G}$ of the operator $\operatorname{tr}\left(\mathscr{D}_{\mu}^{W} \Phi^{\dagger} \mathscr{D}_{\mu}^{W} \Phi\right)$, which is the only one that contributes to the $\zeta_{0}$ kinetic term (while $\operatorname{tr}\left(\mathscr{D}_{\mu}^{W} U^{\dagger} \mathscr{D}_{\mu}^{W} U\right)$ does not). Upon approaching the critical model, $(\rho, \eta) \rightarrow\left(\rho_{c r}, \eta_{c r}\right)$, the canonically normalized $\zeta_{0}$ field has a squared mass $m_{\zeta^{0}}^{2} \sim\left|\hat{\mu}_{\Phi}^{2}\right| /(1-\bar{\gamma}) \rightarrow+\infty$. Decoupling of the $\zeta_{0}$ field in the critical model limit can easily be shown to imply $|\tilde{C}| \leq O\left((1-\bar{\gamma})^{1 / 2}\right)^{\rho \rightarrow \rho_{c r}, \eta \rightarrow \eta_{c r}} 0$ for the coefficient $\tilde{C}$ of an otherwise expected term $\tilde{C} \Lambda_{S} R \operatorname{tr}\left(\mathscr{D}_{\mu}^{W} U^{\dagger} \mathscr{D}{ }_{\mu}^{W} U\right)$ in $\Gamma_{4, c r}^{N G}$.

Remark V) The occurrence in $\Gamma_{4, c r}^{N G}$ of the NP terms $\propto C_{1}, C_{2}$ is reflected in the presence of NP terms in the r.h.s. of the effective (renormalized) SDE associated to $\tilde{\chi}_{L, R}$ transformations, e.g.

$$
Z_{\tilde{J}} \partial_{\mu} \tilde{J}_{\mu}^{L, i}=C_{1} \Lambda_{S}\left(\bar{Q}_{L} \frac{\tau^{i}}{2} U Q_{R}-\text { h.c. }\right)+C_{2} \Lambda_{S}^{2} i g_{W} \operatorname{tr}\left(\Phi^{\dagger}\left[\frac{\tau^{i}}{2}, W_{\mu}\right] \mathscr{D}_{\mu}^{W} \Phi+\text { h.c. }\right) .
$$

Since the $\tilde{\chi}_{L, R}$ currents have vanishing anomalous dimension in the critical model, as it follows from the fact that for $\hat{\mu}_{\phi}^{2}>0$ they are conserved up to $\mathrm{O}\left(\Lambda_{U V}^{-2}\right)$, the r.h.s. of the effective SDE must be RGI in both the Wigner and the NG phase. In the latter case we conclude that the operators $C_{1, Q}\left[\bar{Q}_{L} U Q_{R}+\right.$ h.c. $]$ and $C_{2} \operatorname{tr}\left(\mathscr{D}_{\mu}^{W} U^{\dagger} \mathscr{D}_{\mu}^{W} U\right)$ in $\Gamma_{4, c r}^{N G}$, as well as their $\tilde{\chi}$-variations in the SDE, are RGI and UV-finite - just as it would happen for ordinary soft mass terms put by hand. This implies that the dimensionless coefficients $C_{1, Q}$ and $C_{2}$ are non-trivial functions of the various bare couplings (starting to $\mathrm{O}\left(g_{S}^{4}\right)$ or higher in $g_{S}$ ) and are endowed with a $\Lambda_{U V}$-dependence that compensates for the one of $\left[\bar{Q}_{L} U Q_{R}+\right.$ h.c. $]$ and $\operatorname{tr}\left(\mathscr{D}_{\mu}^{W} U^{\dagger} \mathscr{D}_{\mu}^{W} U\right)$, respectively.

The model (2.1) is power counting renormalizable and the condition (2.8) of maximal restoration of $\tilde{\chi}$ symmetries fixes completely the parameters $\rho$ and $\eta$. If one were replacing $\mathscr{L}_{W i l}$ in the UV-regulated Lagrangian by some other set of $\tilde{\chi}$ violating $d>4$ terms (respecting all the exact symmetries of $\mathscr{L}_{\text {toy }+\mathrm{W}}$ ), the condition of maximal $\tilde{\chi}$ symmetry restoration would fix only $\eta_{c r}$ and one combination of the coefficients in front the various $\tilde{\chi}$ violating $d>4$ terms, but the low energy physics would stay unchanged. Even in the presence of "NP anomalies" to full $\tilde{\chi}$ symmetry restoration in the NG phase, the low energy physics is expected to be controlled by the renormalized gauge couplings, the number of fermions and their transformation properties under the gauge groups. Universality and predictive power are in this sense preserved within the class of UV reg-

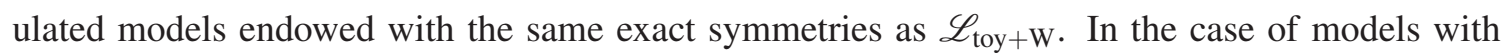
several types and/or generations of fermions, low energy physics will in general also depend on the 
ratios of the critical parameters for the $\tilde{\chi}$ violating terms in the various fermion sectors.

\section{Conclusions and outlook}

Based on the numerical evidence of Ref. [7] in favour of the elementary mass generation mechanism of Ref. [5], we have discussed the key features of the model (2.1), which in general carry over to possible extensions of phenomenological interest and can be summarised as follows. A) The principle of maximal restoration of the (broken) $\tilde{\chi}_{L} \times \tilde{\chi}_{R}$ symmetry ensures both naturalness of the effective masses and the appropriate form of universality that holds at the NP level in $g_{S}^{2}$.

B) The "NP anomaly" giving mass to the $Q$-fermions is also responsible for the NP dynamical mass of weak gauge bosons, which absorb the elementary NG-bosons present in the $g_{W}=0$ limit.

A lot remains to be done to move towards more realistic theories with elementary particle masses generated by "NP anomalies" as discussed above. First, the model (2.1) should be extended to three generations of quark and leptons, while the full EW interaction has to be included by promoting the exact $\chi_{L} \times U(1)_{Y}$ invariance to a gauge symmetry. Most importantly, a "Tera-strong" force and a set of "Tera-fermions" (name coming from Ref. [13]) that communicate with ordinary matter (quarks or leptons) via strong and/or EW interactions [10] should be also included in the model. The "Tera-strong" force is a new non-Abelian gauge interaction that becomes strong at the scale $\Lambda_{T} \gg \Lambda_{Q C D}$, where $\Lambda_{T}$ can be roughly estimated to lie in the few TeV range [5, 10], if the experimental masses of the top quark and weak gauge bosons have to be reproduced - see Eq. (2.11) with $\Lambda_{S}$ replaced by $\Lambda_{T}$. As the condition of $\tilde{\chi}$ restoration entails the decoupling of the $\zeta_{0}$ component of the basic scalar field $\Phi$, one ends up with models of the Composite Higgs [14] type, where the Higgs boson is a bound state $[5,10]$ in the $W W+Z Z$ channel that gets formed owing to the exchange of "Tera-meson" resonances between two weak gauge bosons.

\section{References}

[1] G. 't Hooft, NATO Sci. Ser. B 59 (1980) 135.

[2] C. Froggatt and H. Nielsen, Nuclear Physics B 147 (1979) 277.

[3] W. A. Bardeen, C. T. Hill and M. Lindner, Phys. Rev. D 41 (1990) 1647.

[4] A. Hasenfratz, P. Hasenfratz, K. Jansen, J. Kuti and Y. Shen, Nucl. Phys. B 365 (1991) 79.

[5] R. Frezzotti and G. C. Rossi, Phys. Rev. D 92 (2015) 054505.

[6] S. R. Coleman and E. J. Weinberg, Phys. Rev. D 7 (1973) 1888.

[7] S. Capitani et al., Lattice 2018, East Lansing, MI, United States, July 22-28, 2018.

[8] K. G. Wilson, Phys. Rev. D 10 (1974) 2445.

[9] L. H. Karsten and J. Smit, Nucl. Phys. B 183 (1981) 103.

[10] R. Frezzotti and G. C. Rossi, work in preparation .

[11] E. Witten, Phys. Lett. B117 (1982) 324.

[12] M. F. L. Golterman and D. N. Petcher, Phys. Lett. B225 (1989) 159.

[13] S. L. Glashow, in 11th Intern.l Workshop on Neutrino Telescopes, Venezia, February 22-25, 2005.

[14] E. g. for a recent review see: G. Panico and A. Wulzer, Lect. Notes Phys. 913 (2016) pp.1. 